selective amestic effect on episodic memory in children without significantly impairing long-term memory or attention.

Lorazepam has become increasingly popular for the treatment of status epilepticus in children; the usual median dose is $0.1 \mathrm{mg} / \mathrm{kg}$. Midazol am, a benzodiazepine used primarily for induction of anesthesia, in contrast to lorazepam and diazepam, is water soluble and its injection is neither painful nor irritating by the intramuscular route. Midazolam $15 \mathrm{mg}$ IM was as effective in abolishing spikes as $20 \mathrm{mg}$ of diazepam IV five minutes after administration in a study in adults with epilepsy. (Jawad S et al. J Neurol Neurosurg Psychiat 1986; 49:1050). The half life of lorazepam is longer than that of diazepam or midazolam, however, and its duration of action is more prolonged.

\title{
CARBAMAZEPINE THERAPY AND LONG-TERM PROGNOSIS OF EPILEPTIC CHILDREN
}

The long term prognosis in 90 children with partial or generalized tonic-clonic seizures treated with $\mathrm{CBZ}$ has been evaluated in the Department of Pediatrics, Kyoto University, Kyoto, Japan. Sixty-seven (74\%) treated with CBZ monotherapy were seizure free for more than three years. Fifty (56\%) had no epileptiform discharge on the follow-up EEG. Patients with mental retardation and a genetic predisposition were more likely to have an abnormal EEG. The incidence of mental retardation was significantly higher in those treated with polytherapy. The prognosis of patients with partial seizures secondarily generalized was less favorable than that of the other patients. Patients without mental retardation more often received CBZ monotherapy and patients with seizures of undetermined etiology more often received polytherapy. The lowest blood level of $\mathrm{CBZ}$ for maintenance was $4 \mathrm{meg} / \mathrm{ml}$ and maximum blood levels ranged from 6-12 meg/ml. Side effects were observed in 20 patients who had drowsiness, 4 ataxia, 2 a rash and 1 had anorexia. The SGOT, SGPT, or both were elevated in 16 patients. Leukopenia between 2,000 and 4,000 occurred in 32 patients. (Okuno $T$ et al. Carbamazepine therapy and long-term prognosis in epilepsy of childhood. Epilepsia Jan/Feb 1989; 30:57-61).

COMMENT. There was no correlation between the type of seizure and the prognosis of the patients in this study. All patients with simple partial seizures and benign epilepsy of children with centrotemporal foci were seizure free for more than one year and the majority were seizure free for more than three years. There was no correlation between a history of febrile convulsions and the prognosis of children with partial or generalized tonic-clonic seizures. Patients with partial seizures secondarily generalized had a less favorable prognosis than that of other patients.

SEIZURES IN OFFSPRING OF EPILEPTIC PARENTS

The risks of unprovoked seizures in the offspring of parents with generalized versus partial epilepsies among 687 patients born in Rochester, MN between 1922 and 1985 and followed for the occurrence of seizures through 1986 are reported from the Division of Epidemiology and Department of Neurology, Columbia University, New York, the University of Texas Health 